\title{
An unusual case of resistant cough
}

\author{
DPD Wijesinghe, S Ethulgama, DRS Adicaram Wijesinghe
}

Abstract

Little is known about the prevalence of dissociative disorders in children. Identification of atypical presentations of dissociative disorder in children maybe a challenge, and could lead to misdiagnosis and delay in treatment. Here we present an unusual example of a child with grief and dissociative symptoms, following the loss of a pet dog.

Key words: dissociative disorder, loss of pet

SL J Psychiatry 2014; 5(2) 32-34

\section{Case report}

A 12-year old boy presented with a non-productive cough of 6-months duration. There was no fever, and the cough was absent during sleep. He had initially been treated by a family physician with antibiotics, but the symptoms increased. Thereafter, he had been seen by a pediatrician, a respiratory physician, and an ENT surgeon. Examination of the respiratory system was normal, as were all investigations, including chest X-ray, full blood count, and ESR. The Mantoux test was negative. The patient had been prescribed more antibiotics, as well as antihistamine and anti spasmodic medication, with poor response. Due to persisting symptoms, he was subsequently referred to psychiatric services.

In the history it was revealed that the child's pet dog had died two weeks before the onset of his symptoms. Subsequently the child was noted to be very distressed, weeping and preoccupied with his dead pet. He had dreamt about the dog, and had requested the parents to find him another dog, which the parents had refused. He was the only child in the family and was attached to both parents, especially his father. He was a quiet, pleasant child with a few selected friends. The child was of average intelligence.

On examination it was observed that the child had episodic bouts of coughing. It was atypical in sound and form. On further examination, it became clear that the child was making the sound of a dog barking. The child did not seem to be aware that he was barking, and did not appear to be consciously imitating a bark. There was no attention seeking behavior and there was no evidence of change of intensity of symptoms associated with attention by others. The child appeared distressed. The child was diagnosed to be suffering from a dissociative disorder with pathological grief.

The child was encouraged to talk openly and express emotions about the death of the pet dog. The child was reassured and the possibility of getting another pet dog was discussed. After one week, the child was well without the 'cough' and was much less distressed.

\section{Discussion}

\section{Differential diagnosis}

The most probable diagnosis in this child was dissociative disorder with pathological grief. Psychogenic cough with pathological grief, and Gilles de la Tourette syndrome were also considered as differential diagnosis.

Facing the death of a pet could be a traumatic event during childhood. Grief due to loss of a pet is natural. Most of adults may remember the death of their pets during childhood. A loss of a pet may be the first experience of bereavement for a child. The child may feel depressed, with a sense of emptiness, sadness, guilt and anger at friends whose pets are still alive. They also may develop sleep problems, somatic symptoms, irritability and unexplained physical symptoms. The child may wonder what will happen after the death. There is much literature available about grief reactions due to bereavement of a spouse, children, parents, sibling and friends, but only few reports available regarding pathological grief in children due to loss of a pet.

In the DSM 5 pathological grief is included as a 'Persistent Complex Bereavement Disorder'. The proposed diagnostic criteria state that in young children, yearning may be expressed in play and behaviour, including behaviours that reflect separation and/or reunion with the lost attachment figure (1). The ICD 10 has a special category to diagnose abnormal grief in F43.20 to 43.25 (2).

Dissociative disorder is described in both DSM 5 and ICD $10(1,2)$. In the ICD10, this is classified as a trance and possession disorder (F44.3), under the subtype 'Transient dissociative [conversion] disorder occurring in childhood and adolescence' (F 44.82) (2).

The differentiation of dissociative disorder from psychogenic cough in this child was a challenge. Psychogenic cough can be precipitated by various emotional stimuli. It has well defined characteristics and 
is usually croupy, barky or explosive in nature. It is absent during sleep. A typical 'chin on chest' posture with the hand held to neck while coughing is also characteristic (3). Our patient did not show these features associated with cough.

Gilles de la Tourette syndrome was also considered as a differential diagnosis, since patients may present with respiratory tics, which appear similar to a cough. Respiratory tics in Tourette syndrome may mimic a 'barking cough'. It is extremely rare and is described as the barking cough of puberty (4). This patient did not have any other features of Gilles de la Tourette syndrome such as eye blinking, facial grimacing, echolalia, coprolalia or other tics, and the cough like noise in this patient was very similar to the barking vocalisation of a dog. Therefore Gilles de la Tourette syndrome was considered unlikely in this patient.

To our knowledge childhood grief associated with dissociative features after the loss of a pet, has not been described previously in the medical literature. However grief in adults, after the loss of a pet, has been documented. One study of 207 volunteers investigated antecedents of human grief response associated with the loss of pet; and reported that the level of attachment to the deceased pet, the perceived understanding from others, and presence of other stressful events, significantly predicted the nature of the grief response (5). Another study conducted in affected regions of Hurricane Katrina found that pet loss was strongly associated with depression, acute stress and post traumatic stress disorder (6).

Dissociative symptoms mimicking animal behaviours have also been described previously, though less commonly. In one previous report, a 13-year old girl developed barking vocalisation and shaking movements for four weeks after a viral infection of the upper respiratory tract. She was diagnosed to be suffering from a dissociative disorder and recovered with treatment (7). Harry and Goldwasser described a case of 'barking' in a 35 year old man from Southeastern California. The patient was diagnosed to be having dissociative disorder and recovered after therapeutic intervention (8).

In Sri Lanka, some believe that persons suffering from human rabies show symptoms of barking and other doglike behaviours. This belief may have arisen in the context of dissociative behaviours or delirium, in those affected by the disease, particularly in the past when human rabies was more common.

\section{Coping with the loss of a pet}

Children below ten years usually do not have a clear concept of death. Grief due to a loss of a pet is an opportunity to teach a child about coping with grief and loss. It is best not to shield the child from the fact that the pet is dead, since this may ultimately cause the child to become more confused and frightened. The child should be allowed to talk about the loss and to ventilate his emotions. The child should be involved in the process of the funeral of the pet (9). Rituals may help the child to come to terms with the loss - for example, rituals such as planting a tree in memory of the pet, making a photo album, or lighting a candle. Rituals after the death of pets are not uncommon in Sri Lanka. Funeral activities, giving of alms on behalf of the lost pet, and the making of monuments are some the rituals seen in Sri Lanka.

The issue of whether or not the child should get another pet is not a simple individual choice. The child should not be pushed to get a new pet in order to get over from the problem. One pet will not replace another. It may even lead to resentment or maltreatment of the new pet. If the child is able to express grief about the lost pet, and shows a desire for a new pet, then the child could be offered another pet.

\section{Conclusion}

Dissociative disorder is not uncommon in children but little is known about the prevalence. Precipitating factors may include all types of life stressors. Misdiagnosis is possible when the presentation is atypical and uncommon as in this presentation of dissociative barking. Death of a pet is a traumatic event for a child. Grief due to death of a pet can give rise to varied psychological problems including dissociative disorders. There is growing interest about ways of coping with the death of a pet. Some of these ways of coping have existed for many years in the Sri Lankan culture.

\section{Declaration of interest}

None declared

DPD Wijesinghe, National Institute of Mental Health, Angoda, Sri Lanka

S Ethulgama, Sirimawo Bandaranayake Childrens Hospital Peradeniya, Sri Lanka

DRS Adicaram Wijesinghe, Institute of Legal Medicine and Toxicology, Colombo, Sri Lanka

Corresponding author: DPD Wijesinghe

Email: wijewijewije@yahoo.com

\section{References}

1. American Psychiatric Association (APA). Diagnostic and Statistical Manual of Mental Disorders. $5^{\text {th }}$ ed. APA; Washington, DC; 2013.

2. World Health Organization. International Classification of Mental and Behavioral Disorders. 10 ${ }^{\text {th }}$ ed. Geneva; 1992.

3. Shuper A, Mukamel M, Minouni K, Lerman M, Varsano I. Psychogenic Cough. Arch Dis in Child. 1983; 58(9): 745-7. 
4. Bemstein L. A respiratory tic; "the barking cough of puberty" - report of a case treated successfully. Laryngoscope. 1963; 73(3): 489-98.

5. Goose GH, Barner MJ. Human grief reaction from death of a pet. Anthrozoos 1994; 7(2): 103-12.

6. Hunt M, Al-Awadi H, Johnson M. Psychological sequelae of pet loss following Hurricane Katrina. Anthrozoos 2008; 21(2): 109-21.
7. Hong, J, Schanwald A, Stein T. Barking vocalization and shaking movements in a 13-year old girl. J Dev Behav Pediatr. 2008; 29(2): 135-7.

8. Harry D, Goldwasser MD. Barking man: Case study of rootwork in psychiatry. Jefferson Journal of Psychiatry 1991; 9(1): 82-8.

9. Kenneth RK, Nathaniel DK. And then the dog died. Death Stud. 2006; 30(1): 61-76. 